\title{
ON AN INVERSION THEOREM OF MÖBIUS
}

\author{
J. H. LOXTON and J. W. SANDERS
}

(Received 10 August; revised 3 December 1979)

Communicated by A. J. van der Poorten

\begin{abstract}
The theme of the paper is a Möbius inversion principle for infinite sums. We deal with the origins and unprincipled use of this idea in the nineteenth century, its rigorous justification under minimal hypotheses and some applications to a problem in numerical integration.
\end{abstract}

1980 Mathematics subject classification (Amer. Math. Soc.) : 10 A 20, 65 D 30.

1. The Möbius function

\section{Introduction}

$\mu(n)=\left\{\begin{array}{l}1 \text { if } n=1, \\ (-1)^{k} \text { if } n=p_{1} p_{2} \ldots p_{k} \text { with } p_{1}, p_{2}, \ldots, p_{k} \text { distinct primes, } \\ 0 \text { if } n \text { is divisible by the square of a prime }\end{array}\right.$

occurs implicitly in Euler (1748), paragraph 269, in the reciprocal formulae

$$
\zeta(s)=\sum_{n=1}^{\infty} \frac{1}{n^{s}}=\prod_{p}\left(1-\frac{1}{p^{s}}\right)^{-1}, \frac{1}{\zeta(s)}=\prod_{p}\left(1-\frac{1}{p^{s}}\right)=\sum_{n=1}^{\infty} \frac{\mu(n)}{n^{s}} .
$$

However, its arithmetical significance was first realized by Möbius (1832) with the discovery of a number of inversion formulae. Among these is the classical Möbius inversion formula

$$
g(n)=\sum_{d \mid n} f(d) \leftrightarrow f(n)=\sum_{d \mid n} \mu(d) g(n / d)
$$

(although this simple formulation was given first by Dedekind (1857), page 21, and Liouville (1857)). The formula (2) exemplifies the combinatorial aspect of the 
Möbius function. In this direction, Rota (1964) and his co-workers have developed a general theory of Möbius functions on partially ordered sets which includes the principles of inclusion-exclusion and Möbius inversion as special cases. This work has found many applications in enumeration problems. On the other hand, the inversion problems considered by Möbius have a different and considerably more subtle character. The present work is concerned with the analytic questions provoked by Möbius' formulae and some of their applications.

Our investigations began with the following question in numerical integration : Is it possible to characterize the functions which are integrated exactly by a given rule of approximate integration? For certain common integration rules, this problem can be attacked by Möbius inversion. However, this depends rather delicately on the nature of the integration rule being considered. For this reason, we have arranged the analysis in three sections relating respectively to the trapezoidal rule, the midpoint rule and Simpson's rule.

2. Möbius (1832) raised the following question : Given an 'arbitrary' function $F(z)$ and a function $G(z)$ of the shape

$$
G(z)=\sum_{n=1}^{\infty} a_{n} F\left(z^{n}\right)
$$

express $F(z)$ in terms of the functions $G\left(z^{n}\right)$, say

$$
F(z)=\sum_{n=1}^{\infty} b_{n} G\left(z^{n}\right)
$$

If $F(z)$ is a power series, say $F(z)=c_{1} z+c_{2} z^{2}+\ldots$, we can treat this as a question in formal power series and it is easily seen that the coefficients $b_{n}$ are obtained from the given coefficients $a_{n}$ by the formulae

$$
\sum_{d / n} a_{d} b_{n / d}= \begin{cases}1 & \text { if } n=1 \\ 0 & \text { if } n>1\end{cases}
$$

For given $a_{n}$ with $a_{1} \neq 0$, this determines the $b_{n}$ uniquely. Equivalently, and more enlighteningly, (4) can be written in terms of formal Dirichlet series

$$
\sum_{n=1}^{\infty} \frac{a_{n}}{n^{s}} \cdot \sum_{n=1}^{\infty} \frac{b_{n}}{n^{s}}=1 ;
$$

that is, the $a_{n}$ and the $b_{n}$ form Dirichlet-inverse sequences. In particular, if $a_{n}=1$ for all $n$, then $b_{n}=\mu(n)$ by (1) and we have, at least formally,

$$
G(z)=\sum_{n=1}^{\infty} F\left(z^{n}\right) \leftrightarrow F(z)=\sum_{n=1}^{\infty} \mu(n) G\left(z^{n}\right) .
$$

Further specialization gives the equations

$$
\frac{z}{1-z}=\sum_{n=1}^{\infty} z^{n}, \quad z=\sum_{n=1}^{\infty} \mu(n) \frac{z^{n}}{1-z^{n}} .
$$


The following are also easy deductions from the inversion principle embodied in (3) and ( 3 bis) :

$$
\log _{-1} \frac{1}{1-z}=\sum_{n=1}^{\infty} \frac{z^{n}}{n}, \quad z=\sum_{n=1}^{\infty} \frac{\mu(n)}{n} \log \frac{1}{1-z^{n}}
$$

and

$$
\arctan z=\sum_{n=1}^{\infty} \frac{\varepsilon(n) z^{n}}{n}, \quad z=\sum_{n=1}^{\infty} \frac{\varepsilon(n) \mu(n)}{n} \arctan z^{n},
$$

where $\varepsilon(n)$ is the nonprincipal character modulo 4 , that is

$$
\varepsilon(n)= \begin{cases}1 & \text { if } n \equiv 1 \quad(\bmod 4) \\ -1 & \text { if } n \equiv-1 \quad(\bmod 4) \\ 0 & \text { if } n \text { is even. }\end{cases}
$$

Möbius makes highly ingenious and unprincipled use of these results. Of course, despite the thunderings of Cauchy and Abel, these arguments are very much in the spirit of the time. Thus, in (5), he writes $z=1-w$ and $1-z^{n}=n w$, that is $w$ is an 'infinitesimal', and so obtains

$$
1-w=\sum_{n=1}^{\infty} \frac{\mu(n)}{n} \cdot \frac{1}{w} .
$$

After multiplying through by $w$, this yields

$$
\sum_{n=1}^{\infty} \frac{\mu(n)}{n}=0 .
$$

Euler (1748), paragraph 277, had already obtained this result by taking $s=1$ in (1), but (8) is loosely speaking equivalent to the prime number theorem and so a rigorous proof had to await the discoveries of Hadamard and de la Vallée Poussin. Treating (6) in the same way Möbius obtained

$$
e^{1-w}=\prod_{n=1}^{x}(n w)^{-\mu(n) / n} .
$$

$\mathrm{By}(8)$, the product of the powers of $w$ on the right is 1 , so he has the beautiful formula

$$
e=2^{1 / 2} 3^{1 / 3} 5^{1 / 5} 6^{-1 / 6} 7^{1 / 7} 10^{-1 / 10} 11^{1 / 11} 13^{1 / 13} 14^{-1 / 14} 15^{-1 / 15} \ldots,
$$

or more prosaically,

$$
\sum_{n=1}^{\infty} \frac{\mu(n) \log n}{n}=-1
$$

Again, this is intimately connected with the prime number theorem. (The relations between the prime number theorem and ( 8 ) and $(8$ bis) were first made clear by 
Landau (1899).) Finally he sets $z=1$ in (7) to give

$$
\frac{4}{\pi}=\sum_{n=1}^{\infty} \frac{\varepsilon(n) \mu(n)}{n}=\prod_{p}\left(1-\frac{\varepsilon(p)}{p}\right) .
$$

Euler (1748), paragraph 285, obtained this by formal manipulation of Dirichlet series.

The equations ( 3 ) and ( 3 bis) take a more convenient form if we set $F\left(e^{z}\right)=f(z)$ and $G\left(e^{2}\right)=g(z)$; formally

$$
g(z)=\sum_{n=1}^{\infty} a_{n} f(n z) \leftrightarrow f(z)=\sum_{n=1}^{\infty} b_{n} g(n z) .
$$

The particular case

$$
g(z)=\sum_{n=1}^{\infty} f(n z) \leftrightarrow f(z)=\sum_{n=1}^{\infty} \mu(n) g(n z)
$$

has a curious history and a number of rediscoverers.

Tchebychef(1851) derived several special cases of (9), including Möbius' formulae (5), (6) and (7). His argument is still formal; essentially, he assumes an inversion theorem of the shape (9) and deduces the relation (4) between the coefficients $a_{n}$ and $b_{n}$ by taking $f(z)=z^{-s}$. Given this, he is careful to follow higher standards of rigour and his conclusions are less scintillating than Möbius' work described above. This paper dates from the period in which Tchebychef worked on problems concerning the distribution of prime numbers and the idea of Möbius inversion plays an important role in his memoir of 1852 . If

$$
T(x)=\sum_{n \leqslant x} \log n, \quad \psi(x)=\sum_{p^{m} \leqslant x} \log p
$$

as usual, then Tchebychef's basic identity is

$$
T(x)=\sum_{n=1}^{\infty} \psi(x / n) .
$$

Applying Möbius inversion gives

$$
\psi(x)=\sum_{n=1}^{\infty} \mu(n) T(x / n) .
$$

Although accurate estimates are available for $T(x)$, this formula cannot be used to estimate $\psi(x)$ because of the erratic behaviour of the coefficients $\mu(n)$. Tchebychef succeeded in obtaining bounds for $\psi(x)$ by using an approximate Möbius inversion formula. This was later refined by Selberg in his elementary proof of the prime number theorem. It is interesting to note that Riemann (1859) also derives his approximation

$$
\pi(x)=\operatorname{li} x-\frac{1}{2} \operatorname{li} x^{1 / 2}-\frac{1}{3} \operatorname{li} x^{1 / 3}-\frac{1}{5} \operatorname{li} x^{1 / 5}+\frac{1}{6} \operatorname{li} x^{1 / 6}-\ldots
$$


for the number of primes not exceeding $x$ from a variant of the Möbius inversion formula, namely

$$
G(z)=\sum_{n=1}^{\infty} \frac{1}{n} F\left(z^{1 / n}\right) \leftrightarrow F(z)=\sum_{n=1}^{\infty} \frac{\mu(n)}{n} G\left(z^{1 / n}\right) .
$$

Bachman (1894), pp. 310 to 311, also was apparently unaware of Möbius' paper. His argument for the inversion formula (10) runs as follows : If

$$
g(z)=\sum_{n=1}^{\infty} f(n z)
$$

then

$$
\sum_{n=1}^{\infty} \mu(n) g(n)=\sum_{n=1}^{\infty} \mu(n) \sum_{m=1}^{\infty} f(m n)=\sum_{k=1}^{\infty} f(k) \sum_{n j k} \mu(n)=f(1),
$$

where we have used (4) with $a_{n}=1$ and $b_{n}=\mu(n)$ for the last step. Possible difficulties involved with changing the order of summation of the double series here are not considered. Bachman goes on to apply this particular inversion theorem to obtain a number of formulae for the arithmetic functions of elementary number theory. In fact, convergence questions are irrelevant in this work and in the applications to prime numbers since the series always degenerate to finite sums. Bachman's derivation of the inversion theorem is justified if the double series which occurs above is absolutely convergent. The general case can be handled in the same way, giving the result recorded below. Surprisingly, the first rigorous treatment of these matters appears to be the papers of Hille and Szasz (1936) and Hille (1937).

THEOREM 1. If $a_{n}$ and $b_{n}$ are related by (4),

$$
g(n)=\sum_{m=1}^{\infty} a_{m} f(m n) \quad(n=1,2,3, \ldots),
$$

and the double series $\sum_{k, m} a_{k} b_{m} f(k m n)$ is absolutely convergent, then

$$
f(n)=\sum_{m=1}^{\infty} b_{m} g(m n) \text {. }
$$

The theorem leads easily to the following symmetrical version of $(10)$.

COROLlary. The following two assertions are equivalent :

$$
g(n)=\sum_{m=1}^{\infty} f(m n) \text { for } n=1,2,3, \ldots
$$

and

$$
\begin{gathered}
\sum_{n=1}^{\infty} n^{\varepsilon}|f(n)|<\infty \quad \text { for some } \varepsilon>0 \\
f(n)=\sum_{m=1}^{\infty} \mu(m) g(m n) \quad \text { for } n=1,2,3, \ldots
\end{gathered}
$$


and

$$
\sum_{n=1}^{\infty} n^{\varepsilon}|g(n)|<\infty \text { for some } \varepsilon>0
$$

Hille and Szasz (1936) also point out the need for caution here by giving a counterexample to the truth of (10) for general functions $f$ and $g$. If $g(n)=1 / n$, then $f(n)=\sum_{m} \mu(m) g(m n)$ is identically zero, as we have seen, so that $g(n) \neq \sum_{m} f(m n)$. In the other direction, if $f(n)=\mu(n) / n$, then $g(n)=\sum_{m} f(m n)$ is identically zero and again (10) fails.

\section{Inversion of the trapezoidal rule}

3. Let $f$ be a continuous function on the closed interval $[0,1]$. According to the trapezoidal rule of quadrature, the integral of $f$ over $[0,1]$ is to be approximated by

$$
T_{n} f=\frac{1}{n}\left\{\frac{1}{2} f(0)+f\left(\frac{1}{n}\right)+\ldots+f\left(\frac{n-1}{n}\right)+\frac{1}{2} f(1)\right\} \quad(n=1,2, \ldots) .
$$

How much information is contained in the sequence $\left\{T_{n} f\right\}$ of approximate integrals of $f$ ?

The Fourier expansion of $f$ is

$$
f(t)=\sum_{n=-\infty}^{\infty} \hat{f}(n) e^{2 \pi i n t}, \text { with } \hat{f}(n)=\int_{0}^{1} f(t) e^{-2 \pi i n t} d t,
$$

the Fourier series being Cesàro summable to $f(t)$ for $0<t<1$ and to $\frac{1}{2}\{f(0)+f(1)\}$ at $t=0$ and 1 . Consequently, the approximate integrals $T_{n} f$ can be expressed in terms of the Fourier coefficients by

$$
T_{n} f=\sum_{m=-\infty}^{\infty} \hat{f}(m n) \quad(C, 1),
$$

the series being Cesàro summable. If the Fourier series is convergent and not merely Cesàro summable, then the sums (11) also converge. Under suitable conditions, the Möbius inversion principle (10) can be used to invert (11) as follows :

$$
\hat{f}(n)+\hat{f}(-n)=\sum_{m=1}^{\infty} \mu(m) \Delta_{m n} f, \quad \text { where } \Delta_{n} f=T_{n} f-\int_{0}^{1} f(t) d t .
$$

Lyness (1970) has developed this idea into a practical numerical procedure for calculating Fourier coefficients.

It is easy to see that the trapezoidal rule is exact for linear functions and for functions which are odd about $\frac{1}{2}$, that is $\Delta_{n} f=0$ for all $n$ when $f$ is a function of one of these types. We used the above ideas to obtain a partial converse to these observations (Loxton and Sanders (1980)). To state this, let us write

$$
f(t)=\hat{f}(0)+\{f(1)-f(0)\}\left(t-\frac{1}{2}\right)+g(t),
$$


where $g$ is continuous and periodic. If the trapezoidal rule is exact for $f$ and the Fourier series of $g$ is absolutely convergent, then $g$ is odd about $\frac{1}{2}$. The proof depends on justifying (12), at least for the special case in which all $\Delta_{n} f$ are zero.

4. We shall discuss the Möbius inversion principle (9) subject to the restriction that the coefficients $a_{n}$ are completely multiplicative, that is $a_{m n}=a_{n} a_{n}$ for all $m$ and $n$, and $a_{1}=1$. The Dirichlet-inverse sequence is then given by $b_{n}=\mu(n) a_{n}$. This is only slightly more general than the inversion problem arising from the trapezoidal rule; indeed, if $a_{n} \neq 0$ for all $n$, then (9) reduces to (10) if we replace $a_{n} f(n)$ and $a_{n} g(n)$ by $f(n)$ and $g(n)$ respectively. We aim to determine as precisely as possible the domain of validity of the inversion principle (9). We begin with a positive result which improves on the corollary to Theorem 1 .

THEOREM 2. Suppose that $a_{n}$ is completely multiplicative and $a_{1}=1$ and that all the series $\sum_{m} a_{m} f(m n)$ and $\sum_{m} a_{m} g(m n)$ are absolutely convergent. Then

$$
g(n)=\sum_{m=1}^{\infty} a_{m} f(m n) \leftrightarrow f(n)=\sum_{m=1}^{\infty} \mu(m) a_{m} g(m n) .
$$

Proof. Consider first the implication from left to right. Let $v(x)$ denote the least common multiple of the positive integers not exceeding $x$ and let $\mathscr{D}(x)$ denote the set of positive integers whose prime factors are all greater than $x$. We have

$$
\sum_{m|v| x \mid} \mu(m) a_{m} g(m n)=\sum_{m \mid v(x)} \mu(m) a_{m} \sum_{l=1}^{\infty} a_{l} f(l m n)=\sum_{k=1}^{\infty} a_{k} f(k n) \sum_{m \mid\langle k, v(x))} \mu(m),
$$

the rearrangement being justified because the sum over $m$ is finite. The inner sum here is zero unless $(k, v(x))=1$, so

$$
\sum_{m \mid v(x)} \mu(m) a_{m} g(m n)=\sum_{k \text { in } /(x)} a_{k} f(k n) .
$$

Now let $x$ tend to infinity. Since the intersection of all the $\mathscr{D}(x)$ is 1 and the series involved are absolutely convergent, we obtain

$$
\sum_{m=1}^{\infty} \mu(m) a_{m} g(m n)=f(n) .
$$

For the converse, let $\mathscr{E}(x)$ be the set of positive integers $k$ with the property that any prime which divides both $k$ and $v(x)$ divides $k$ to a higher power than it divides $v(x)$. Then proceeding as before,

$$
\begin{aligned}
\sum_{m \mid v(x)} a_{m} f(m n)=\sum_{m ; v(x)} a_{m} \sum_{l=1}^{\infty} \mu(l) a_{l} g(l m n) & =\sum_{k=1}^{\infty} a_{k} g(k n) \sum_{n \mid k, v(x))} \mu\left(\frac{k}{n}\right) . \\
& =\sum_{k i n^{\prime}(x)} \mu\left(\frac{k}{(k, v(x))}\right) a_{k} g(k n),
\end{aligned}
$$


and, letting $x$ tend to infinity,

$$
\sum_{m=1}^{\infty} a_{m} f(m n)=g(n)
$$

5. We must now consider the extent to which Theorem 2 is best possible. For simplicity, we discuss only the case $a_{m}=1$; there is no essential loss of generality in doing so.

First, suppose the $f(n)$ are given and the series $\sum f(m)$ is absolutely convergent. If $g(n)$ is defined by $g(n)=\sum_{m} f(m n)$, then the proof of Theorem 2 shows that the $f(n)$ may be recovered from the $g(n)$, without any additional hypotheses, by

$$
f(n)=\lim _{x \rightarrow \infty} \sum_{m / v(x)} \mu(m) g(m n) .
$$

The example at the end of Section 2 already shows that this conclusion cannot hold in general. In fact, we can exhibit functions $f(n)$ for which $\sum_{m} f(m n)=0$ for each $n$ and $\sum|f(m)|$ diverges arbitrarily slowly and so the transformation taking the $f(n)$ to the $g(n)$ cannot be inverted if the hypothesis of absolute convergence of $\sum f(m)$ is weakened.

The example depends on the following theorem of Wintner (1943). Wintner actually proves more, but this special case can be proved more directly than he suggests.

THEOREM 3. Let $\mathscr{P}$ be a set of primes with $\sum_{p \text { in }}(1 / p)$ divergent. Let $\mu_{p}(n)$ be the multiplicative function whose values at the prime powers are given by $\mu_{p}\left(p^{k}\right)=-1$ for $p$ in $\mathscr{P}$ and $k=1$ and $\mu_{p}\left(p^{k}\right)=0$ otherwise. Then $\sum\left(\mu_{p}(n) / n\right)=0$.

ProOf. Let $\mathscr{N}$ be the set of positive integers generated by the primes not in $\mathscr{P}$, let $g(n)$ be the characteristic function of $\mathcal{N}$ and, for convenience, set $h(n)=\mu_{p}(n)$. Note that

$$
\sum_{d \mid n} h(d)=g(n) .
$$

Similarly, let $\mathscr{P}_{m}$ comprise the primes $p$ in $\mathscr{P}$ with $p \leqslant m, \mathscr{N}_{m}$ be the integers generated by the primes not in $\mathscr{P}_{m}, g_{m}(n)$ be the characteristic function of $\mathscr{N}_{m}$ and $h_{m}(n)$ be the Möbius function of $\mathscr{P}_{m}$. Clearly,

$$
g_{m}(n) \leqslant g_{m-1}(n) \quad \text { and } \quad g_{m}(n) \rightarrow g(n) \quad \text { as } \quad m \rightarrow \infty .
$$

By the sieve of Eratosthenes,

$$
\frac{1}{n_{k}=1} \sum_{m}^{n} g_{m}(k) \rightarrow \prod_{p \text { in } \mathscr{s}_{m}}\left(1-\frac{1}{p}\right)
$$

as $n \rightarrow \infty$ and this product tends to 0 as $m \rightarrow \infty$. From this and the monotonic 
convergence in (15), we have

$$
\frac{1}{n} \sum_{k=1}^{n} g(k) \rightarrow 0 \quad \text { as } n \rightarrow \infty .
$$

This states that the series whose $n$th partial sum is $g(n)$ is Cesàro summable and consequently Abel summable to 0 , so we have

$$
(1-r) \sum_{n=1}^{\infty} g(n) r^{n} \rightarrow 0 \quad \text { as } r \rightarrow 1 .
$$

On the other hand, from (14),

$$
\sum_{n=1}^{\infty} g(n) r^{n}=\sum_{n=1}^{\infty} \frac{h(n) r^{n}}{1-r^{n}}
$$

so the series $\sum(h(n) / n)$ is Lambert summable to 0 . Now $h(n)=\mathrm{O}(1)$, so the standard Tauberian theorem of Hardy (1949), Appendix 4, for Lambert summability applies and we conclude that $\sum(h(n) / n)$ is convergent with sum 0 , as required.

Now we can give the example promised above. Let $\mathscr{P}$ be a set of primes with $\sum_{p \text { in },}(1 / p)$ divergent. Take $f(n)=\left(\mu_{p}(n) / n\right)$, where $\mu_{, p}(n)$ is the Möbius function of $\mathscr{P}$, as defined in Theorem 3. We assert that

$$
\sum_{m=1}^{\infty} f(m n)=0 \quad(n=1,2, \ldots) .
$$

If $n$ is divisible by the square of a prime or has a prime factor not in $\mathscr{P}$, then $\mu_{,}(m n)=0$ and (16) is clear. Otherwise, $\mu_{p,}(n) \neq 0$ and we see, by checking values at prime powers, that the multiplicative function $\mu_{\rho p}(m n) / \mu_{, p}(n)$ is just $\mu_{,}(m)$ where $\mathscr{Q}$ is obtained from $\mathscr{P}$ by deleting the primes dividing $n$. Consequently, (16) follows from the theorem applied to the set of primes 2 . Moreover, $\sum|f(m)|$ diverges like

$$
\prod_{p \text { in }}\left(1+\frac{1}{p}\right)
$$

and this rate of divergence can therefore be made arbitrarily slow. More precisely, if $\mathscr{P}$ is suitably regular, we have

$$
\log \sum_{m=1}^{n}|f(n)| \sim \sum_{p \text { in } \mathscr{P}, p \leqslant n} \frac{1}{p} \quad \text { as } n \rightarrow \infty .
$$

To justify this, we note that

$$
\log \sum_{m=1}^{\infty} \frac{|f(m)|}{m^{s}}=\log \prod_{p \text { in } \rho}\left(1+\frac{1}{p^{1+s}}\right) \sim \sum_{p \text { in } \rho} \frac{1}{p^{1+s}} \quad \text { as } s \rightarrow 0 .
$$

Now, if $\mathscr{P}$ is such that the sum on the right of (17) is regularly varying, then (17) follows by means of the standard Tauberian theorem for the Mellin transform. (This is a special case of Hardy (1949), Theorem 108.) 
6. The example of the previous section shows that our discussion of the transform $g(n)=\sum_{m} f(m n)$ must be restricted to functions $f(n)$ for which $\sum f(m)$ is absolutely convergent. The transform can then be inverted by (13), so that the series $\sum_{m} \mu(m) g(m n)$ is at least summable to $f(n)$. We now consider its sum in the usual straightforward sense.

First, the series $\sum_{m} \mu(m) g(m n)$ need not converge, even when $\sum f(m)$ is absolutely convergent. This reflects the fact that the summability method (13) is not regular in the sense of Hardy (1949), Chapter 3. We shall give an example which achieves a little more than the general theory.

Let $\left\{t_{j}\right\}$ be a sequence of positive integers with $t_{j}>2^{t_{j-1}}$ and let $s_{j}$ be the product of the primes between $\frac{1}{2} t_{j}$ and $t_{j}$. Define the function $f(m)$ by $f\left(s_{j}\right)=-2^{-j}$ and $f(m)=0$ elsewhere. Thus $\sum f(m)$ is absolutely convergent. Now set $g(n)=\sum_{m} f(m n)$ and

$$
h(M)=\sum_{m=1}^{M} \mu(m) g(m)=\sum_{k=1}^{\infty} f(k) \sum_{\substack{1 \leqslant m<M \\ m \in k}} \mu(m) .
$$

Consider $h\left(t_{j}\right)$. The term $k=s_{j}$ on the right contributes

$$
-f\left(s_{j}\right)\left\{\pi\left(t_{j}\right)-\pi\left(\frac{1}{2} t_{j}\right)\right\} \sim t_{j} / 2^{j} \log t_{j} ;
$$

the terms with $k>s_{j}$ each contribute $f(k)$, so their sum is $\mathrm{O}(1)$; and, since $s_{i}<t_{j}$ when $i<j$, the terms with $k<s_{j}$ contribute

$$
\sum_{i<j} f\left(s_{i}\right) \sum_{m \mid s_{i}} \mu(m)=0 .
$$

Thus the $h\left(t_{j}\right)$ are unbounded and $\sum \mu(m) g(m)$ does not converge. However, for $n>1$, the series $\sum_{m} \mu(m) g(m n)$ converges to $f(n)$. To see this, observe that

$$
\sum_{m=1}^{M} \mu(m) g(m n)=\sum_{k=1}^{\infty} f(k n) \sum_{\substack{1 \leqslant m \leqslant M \\ m k}} \mu(m)
$$

and the sum over $k$ has at most one nonvanishing term, so the assertion reduces to a finite Möbius inversion.

We can modify this example to give a function $f(n)$ for which $\sum f(m)$ is absolutely convergent, but all the series $\sum_{m} \mu(m) g(m n)$ are divergent. Begin by choosing the sequence $\left\{t_{j}\right\}$ of positive integers with $t_{j}>j ! 2^{t_{j-1}}$ and let $s_{j}$ be the product of the primes between $\frac{1}{2} t_{j}$ and $t_{j}$. Define the function $f_{q}(m)$ by $f_{q}\left(q ! s_{j}\right)=-1 / 2^{j} t_{j-1}$ for $j \geqslant q$ and $f_{q}(m)=0$ elsewhere. Set $g_{q}(n)=\sum_{m} f_{q}(m n)$. By proceeding as before, we find that if $n \mid q$ !, then

$$
\sum_{m=1}^{t_{j}} \mu(m) g_{q}(m n)= \begin{cases}\left\{\pi\left(t_{j}\right)-\pi\left(\frac{1}{2} t_{j}\right)\right\} / 2^{j} t_{j-1}+\mathrm{O}(1) & \text { if } j \geqslant q, \\ \mathrm{O}(1) & \text { if } j<q,\end{cases}
$$

where the implied constants are independent of $n$ and $q$. If $n \nmid q$ !, then $\sum_{m} \mu(m) g_{q}(m n)$ 
converges to $f_{q}(n)$. Finally, let

$$
f(m)=\sum_{q=1}^{\infty} 2^{-q} f_{q}(m) \text { and } g(n)=\sum_{m=1}^{\infty} f(m n) .
$$

The preceding remarks enable us to show that the partial sums

$$
\sum_{m=1}^{t_{j}} \mu(m) g(m n)
$$

are unbounded for every $n$, that is none of the series $\sum_{n} \mu(m) g(m n)$ converges.

Nevertheless, it seems likely that the summability method (13) is at least consistent. More precisely, we conjecture that if $\sum_{m} f(m)$ is absolutely convergent, $g(n)=\sum_{m} f(m n)$ and $\sum_{m} \mu(m) g(m n)$ is convergent, then the value of this last series is $f(n)$.

7. Now we consider some examples relating to the converse direction of Theorem 2. That is, we suppose that the $f(n)$ are defined by $f(n)=\sum_{m} \mu(m) g(m n)$ and we investigate the validity of the inversion $g(n)=\sum_{m} f(m n)$.

We show first that absolute convergence of the series $\sum_{m} \mu(m) g(m n)$ is not enough to give an inversion theorem. Take $g\left(2^{j}\right)=1$ for $j \geqslant 0$ and $g(m)=0$ elsewhere. Then $f(n)=0$ for all $n$, so the inversion theorem fails. For this example, the series $\sum_{m} \mu(m) g(m n)$ even reduces to a finite sum.

Next, let $\mathscr{P}$ be a set of primes with $\sum_{p \text { in },}(1 / p)$ divergent. Take $g(m)=1 / m$ if $m$ is divisible only by the primes in $\mathscr{P}$ and $g(m)=0$ elsewhere. Then $f(n)=0$ for all $n$, by Theorem 3, and again the inversion theorem fails. In this case $\sum_{m} g(m)$ is divergent, but the rate of divergence can be made arbitrarily slow by a suitable choice of $\mathscr{P}$.

So, as before, we must assume that $\sum_{m} g(m)$ is absolutely convergent. The example of the preceding section is easily modified to show that the series $\sum_{m} f(m n)$ need not converge. In fact, if $g(m)=0$ whenever $m$ is divisible by the square of a prime, then

$$
\sum_{m=1}^{M} f(m)=\sum_{k=1}^{\infty} g(k) \sum_{1 \leqslant m \leqslant M} \mu\left(\frac{k}{m}\right)=\sum_{k=1}^{\infty} \mu(k) g(k) \sum_{1 \leqslant m \leqslant M} \mu(m)
$$

which is essentially the same as (18).

\section{Inversion of the midpoint rule}

8. Let $f$ be a continuous function on the interval $[0,1]$. The approximations to the integral of $f$ over this interval determined by the midpoint rule are

$$
M_{n} f=\frac{1}{n}\left\{f\left(\frac{1}{2 n}\right)+f\left(\frac{3}{2 n}\right)+\ldots+f\left(\frac{2 n-1}{2 n}\right)\right\} \quad(n=1,2, \ldots) .
$$


The $M_{n} f$ can be expressed in terms of the Fourier coefficients $\hat{f}$ of $f$ by

$$
M_{n} f=\sum_{m=-\infty}^{\infty}(-1)^{m} \hat{f}(m n) \quad(C, 1),
$$

where, in general, the series is only Cesàro summable. Now, the sequences

$$
a_{n}=(-1)^{n}, \quad b_{n}= \begin{cases}-\mu(n) & \text { for } n \text { odd, } \\ -2^{r-1} \mu(s) & \text { for } n=2^{r} s \text { with } r \geqslant 1 \text { and } s \text { odd }\end{cases}
$$

are Dirichlet inverses, so formal Möbius inversion of (19) gives

$$
\hat{f}(n)+\hat{f}(-n)=\sum_{m=1}^{\infty} b_{m} \Delta_{m n} f, \quad \text { where } \Delta_{n} f=M_{n} f-\int_{0}^{1} f(t) d t .
$$

This inversion no longer fits into the scheme of Theorem 2 and, in fact, its justification requires more stringent conditions as we shall presently show.

The following simple example illustrates the additional problems. Suppose $f(n)=0$ when $n$ is not a power of 2. Define $g(n)$ by $g(n)=\sum_{m}(-1)^{m} f(m n)$, so that $g(n)=0$ when $n$ is not a power of 2 and

$$
g\left(2^{r}\right)=-f\left(2^{r}\right)+\sum_{s=1}^{\infty} f\left(2^{r+s}\right) .
$$

From this,

$$
\sum_{n=1}^{2^{v}} b_{n} g(n)=-g(1)-\sum_{r=1}^{N} 2^{r-1} g\left(2^{r}\right)=f(1)-2^{N} \sum_{r=N+1}^{\infty} f\left(2^{r}\right),
$$

so the inversion $f(1)=\sum_{n} b_{n} g(n)$ is valid if and only if

$$
2^{N} \sum_{r=N+1}^{\infty} f\left(2^{r}\right) \rightarrow 0 \quad \text { as } N \rightarrow \propto \text {. }
$$

In particular, if we take $f\left(2^{r}\right)=2^{-r}$ for $r \geqslant 0$ and $f(n)=0$ elsewhere, then $g(n)=0$ for all $n$ and the postulated inversion fails.

9. We now see how much of an improvement can be made to Theorem 1 in the present case.

THEOREM 4. Define the coefficients $a_{n}$ and $b_{n} b y$

$$
a_{n}=(-1)^{n}, \quad b_{n}= \begin{cases}-\mu(n) & \text { for } n \text { odd }, \\ -2^{r-1} \mu(s) & \text { for } n=2^{r} s \text { with } r \geqslant 1 \text { and } s \text { odd } .\end{cases}
$$

Suppose that the series

$$
\sum_{n=1}^{\infty} f(n) \text { and } \sum_{r=0}^{\infty} \sum_{s \text { odd }} 2^{r} g\left(2^{r} s\right)
$$


are absolutely convergent and that

$$
2^{e} \sum_{r=e+1}^{\infty} f\left(2^{r} n\right) \rightarrow 0 \text { as } e \rightarrow \infty \text { for each } n .
$$

Then

$$
g(n)=\sum_{m=1}^{\infty} a_{m} f(m n) \leftrightarrow f(n)=\sum_{m=1}^{\infty} b_{m} g(m n)
$$

Proof. Let $v(x)$ denote the least common multiple of the odd positive integers not exceeding $x$ and let $\mathscr{D}(x)$ denote the set of positive integers whose prime factors are all greater than $x$. Then

$$
\sum_{m \mid 2^{*} v(x)} b_{m} g(m n)=\sum_{m \mid 2^{\prime} v(x)} b_{m} \sum_{l=1}^{\infty} a_{l} f(l m n)=\sum_{k=1}^{\infty} f(k n) \sum_{m \mid\left(k, 2^{*} v(x)\right)}(-1)^{k / m} b_{m} .
$$

If we set $k=2^{r} s$ and $m=2^{u} v$ where $s, v$ are odd, then the inner sum is

$$
\sum_{v \mid(s, v(x))} \mu(v)\left\{(-1)^{2^{r}+1}+\sum_{u=1}^{\min \{e, r\}} 2^{u-1}(-1)^{2^{r-u}+1}\right\} .
$$

If $(s, v(x))=1$ and $r=0$, this is 1 , while if $(s, v(x))=1$ and $r>e$, it is $-2^{e}$. In all other cases, it is 0 . We therefore have

$$
\sum_{m \mid 2^{e} v(x)} b_{m} g(m n)=\sum_{\sin s /(x)}\left\{f(s n)-2^{e} \sum_{r=e+1}^{\infty} f\left(2^{r} s n\right)\right\} .
$$

Now let $e$ and then $x$ tend to infinity. Our hypotheses imply that $2^{e} \sum_{r>e} f\left(2^{r} s n\right) \rightarrow 0$ as $e \rightarrow \infty$ and that the series $\sum_{s} f(s n)$ and $\sum_{m} b_{m} g(m n)$ are absolutely convergent, so we obtain

$$
\sum_{m=1}^{\infty} b_{m} g(m n)=f(n)
$$

For the converse, let $\mathscr{E}(x)$ be the set of odd positive integers $k$ with the property that any prime which divides both $k$ and $v(x)$ divides $k$ to a higher power than it divides $v(x)$. Then, proceeding as before,

$$
\begin{aligned}
\sum_{m \mid 2^{e} v(x)} a_{m} f(m n) & =\sum_{k=1}^{\infty} g(k n) \sum_{m \mid\left(k, 2^{e} v(x)\right)}(-1)^{m} b_{k / m} \\
& =\sum_{s i n \wedge(x)} \mu\left(\frac{s}{(s, v(x))}\right)\left\{g(s n)+\sum_{r=e+1}^{\infty} 2^{r-e-1} g\left(2^{r} s n\right)\right\}
\end{aligned}
$$

and, letting $e$ and $x$ tend to infinity, we get

$$
\sum_{m=1}^{\infty} a_{m} f(m n)=g(n)
$$

It will be seen from the above proof that each of the separate implications of Theorem 4 is true under slightly weaker hypotheses than the ones we have made. 
However, nothing substantial is salvaged by this restatement. In fact, the necessity for the surprising condition (20) is shown by the example at the end of the previous section. Moreover, we can expect the appearance of the requirements of absolute convergence in the theorem since it effectively includes Theorem 2. For example, if $f(n)$ and $g(n)$ vanish for even integers $n$, the conclusion of the theorem is just

$$
g(n)=\sum_{m=1}^{\infty} f(m n) \leftrightarrow f(n)=\sum_{m=1}^{\infty} \mu(m) g(m n) .
$$

\section{Remarks on some further integration rules}

10. We consider first the higher order Newton-Cotes rules. Let $f(t)$ be a function defined on the interval $[0,1]$. For each positive integer $h$, Lagrange interpolation yields a polynomial $p(t)$ of the shape

$$
p(t)=\sum_{k=0}^{h} L_{k}(t) f\left(\frac{k}{h}\right)
$$

and of degree $h$ which satisfies $p(k / h)=f(k / h)$ for $k=0,1,2, \ldots, h$. Its integral is

$$
\int_{0}^{1} p(t) d t=\sum_{k=0}^{h} w_{h k} f\left(\frac{k}{h}\right)
$$

say, where the numbers $w_{h k}$ are independent of the function $f(t)$. The first few cases are listed in Table 1 from Jordan (1960), p. 513.

TABLE 1

\begin{tabular}{ccccccccc}
\hline$k$ & 0 & 1 & 2 & 3 & 4 & 5 & 6 & \\
\hline 1 & $1 / 2$ & $1 / 2$ & & & & & & $\begin{array}{l}\text { (Trapezoidal rule) } \\
\text { (Simpson's rule) }\end{array}$ \\
2 & $1 / 6$ & $4 / 6$ & $1 / 6$ & & & & & (Boole's formula) \\
3 & $1 / 8$ & $3 / 8$ & $3 / 8$ & $1 / 8$ & & & & \\
4 & $7 / 90$ & $32 / 90$ & $12 / 90$ & $32 / 90$ & $7 / 90$ & & & \\
5 & $19 / 288$ & $75 / 288$ & $50 / 288$ & $50 / 288$ & $75 / 288$ & $19 / 288$ & & \\
6 & $41 / 840$ & $216 / 840$ & $27 / 840$ & $272 / 840$ & $27 / 840$ & $216 / 840$ & $41 / 840$ & \\
\hline
\end{tabular}

Now, if we divide the interval $[0,1]$ into $n$ equal subintervals and use interpolation of degree $h$ on each subinterval, we get the sequence of approximate integrals

$$
I_{n} f=\sum_{j=0}^{n} \delta_{j} \sum_{k=0}^{h} w_{k} f\left(\frac{h j+k}{h n}\right)
$$


where $\delta_{j}=1$ for $1 \leqslant j \leqslant n-1$ and $\delta_{0}=\delta_{n}=\frac{1}{2}$. The previous discussion shows that this integral is exact if $f(t)$ is a polynomial of degree at most $h$. From the symmetry of the situation, the integral is also exact if $f(t)$ is odd about the mid-point of the interval $[0,1]$.

If $f(t)$ is given by its Fourier series

$$
f(t)=\sum_{m=-\infty}^{\infty} \hat{f}(m) e^{2 \pi i m t}, \quad \hat{f}(m)=\int_{0}^{1} f(t) e^{-2 \pi i m t} d t,
$$

then the approximate integrals become

$$
I_{n} f=\sum_{m=-x}^{x} a_{m} \hat{f}(m n) \quad \text { with } a_{m}=\sum_{k=0}^{h} w_{k} e^{2 \pi i k m / h}
$$

The inversion problem can still be solved explicitly for the first few cases of these rules, as we shall now see.

11. Let $q$ be a prime. We consider sequences of coefficients $a_{n}$ which are completely multiplicative except at the prime $q$, that is

$$
a_{m n}=a_{m} a_{n} \text { whenever } q /(m, n) \text { and } a_{1}=1 \text {. }
$$

Such a sequence is determined by giving the values $a_{p}$ at primes $p \neq q$ and the values $a_{q j}=c_{j}$, say, at the powers of $q$. The Dirichlet inverse sequence $b_{n}$ is the multiplicative function given by

$$
b_{n}=\mu(m) a_{m} d_{j} \quad \text { for } n=q^{j} m \text { with } q \mid m,
$$

where

$$
\sum_{i=0}^{j} c_{i} d_{j-i}= \begin{cases}1 & \text { for } j=0 \\ 0 & \text { for } j>0\end{cases}
$$

It will simplify the exposition if we assume that $a_{p}=1$ for primes $p \neq q$; as in Section 4 , this is not really a restriction.

THEOREM 5. Let $q$ be a prime and suppose sequences $a_{n}$ and $b_{n}$ are given $b_{y}$

$$
\left.a_{n}=c_{j} \text { and } b_{n}=\mu(m) d_{j} \text { for } n=q^{j} m \text { with } q\right) m,
$$

where the $c_{j}$ and $d_{j}$ satisfy (21). Choose numbers $\rho_{\infty}$ and $\rho_{0}$ with $1 \leqslant \rho_{x} \leqslant \rho_{0}$ so that the power series $\sum_{j \geqslant 0} c_{j} z^{j}$ is regular in $|z|<\rho_{\infty}^{-1}$ and nonzero in $|z|<\rho_{0}^{-1}$. Suppose there is some $\varepsilon>0$ so that

$$
\begin{gathered}
\sum_{\substack{j, m \\
q \mid m}}\left(\rho_{\infty}+\varepsilon\right)^{j}\left|f\left(q^{j} m\right)\right|<\infty, \\
\left(\rho_{0}+\varepsilon\right)^{e} \sum_{j>e}\left(\rho_{\infty}+\varepsilon\right)^{-e}\left|f\left(q^{j} m\right)\right| \rightarrow 0 \text { as } e \rightarrow \infty \text { for each } m, \text { and } \\
\sum_{\substack{j, m \\
q \mid m}}\left(\rho_{0}+\varepsilon\right)^{j}\left|g\left(q^{j} m\right)\right|<\infty .
\end{gathered}
$$


Then

$$
g(n)=\sum_{m=1}^{\infty} a_{m} f(m n) \leftrightarrow f(n)=\sum_{m=1}^{\infty} b_{m} g(m n) .
$$

ProOF. The idea is the same as in the proof of Theorem 4. For example, if $v(x)$ denotes the least common multiple of the positive integers not exceeding $x$ and prime to $q$ and $\mathscr{D}(x)$ denotes the set of positive integers whose prime factors are all greater than $x$, then we see that $g(n)=\sum_{m} a_{m} f(m n)$ implies

$$
\sum_{m \mid q^{*} v(x)} b_{m} g(m n)=\sum_{s^{\prime \prime \prime}(x)}\left\{f(s n)+\sum_{r>e} f\left(q^{r} s n\right) \sum_{0 \leqslant j \leqslant e} c_{r-j} d_{j}\right\} .
$$

Now $c_{j} \ll\left(\rho_{x}+\varepsilon\right)^{j}$ and $d_{j} \ll\left(\rho_{0}+\varepsilon\right)^{j}$, so letting $e$ and then $x$ tend to infinity in the above yields $f(n)=\sum_{m} b_{m} g(m n)$.

We note that here, in contrast to Theorem 2 , the class of functions $f$ for which the inversion

$$
g(n)=\sum_{m=1}^{\infty} a_{m} f(m n) \rightarrow f(n)=\sum_{m=1}^{\infty} b_{m} g(m n)
$$

is possible cannot be specified in terms of the size of the coefficients $a_{n}$ alone. In fact, the shape of the conditions imposed in Theorem 5 suggests that the simple Theorem 1 may be essentially the best possible inversion theorem for an unrestricted sequence of coefficients $a_{n}$.

12. One example of Theorem 5 has already been discussed in Section 9 . We shall give three more examples taken from the integration rules described in Section 10.

(a) Take $q=2, c_{0}=1$ and $c_{j}=-3$ for $j \geqslant 1$ in Theorem 5. Then $d_{j}=3.4^{j-1}$ for $j \geqslant 1$ and we can take $\rho_{\infty}=1$ and $\rho_{0}=4$. If $f(t)$ is defined on the interval $[0,1]$ and has the Fourier coefficients $\hat{f}(n)$, then

$$
\Delta_{n} f=I_{n} f-\int_{0}^{1} f(t) d t=-\frac{1}{3} \sum_{m=1}^{\infty} a_{m}\{\hat{f}(m n)+\hat{f}(-m n)\},
$$

with $a_{m}$ defined as in Theorem 5 , gives the errors for the approximate integrals of Simpson's rule. We can tidy up the proof of Theorem 5 to obtain the following typical result.

THEOREM 6. Suppose the function $f(t)$ is continuous on the interval $[0,1]$ and its Fourier coefficients $\hat{f}(n)$ satisfy the conditions

$$
\sum_{n}|\hat{f}(n)|<\infty \quad \text { and } \quad 4^{e} \sum_{j>e}\left|\hat{f}\left(2^{j} n\right)\right| \rightarrow 0 \quad \text { as } e \rightarrow \infty \text { for each } n .
$$

If Simpson's rule integrates $f$ exactly, that is $\Delta_{n} f=0$ for all $n$, then $f(t)$ is odd about $\frac{1}{2}$. 
The function $f(t)=\sum_{j \geqslant 0} 4^{-j} \exp \left(2^{j+1} \pi i t\right)$ shows that the second hypothesis in (22) cannot be dropped; it is integrated exactly by Simpson's rule, but is clearly not odd.

(b) Take $q=3, c_{0}=1$ and $c_{j}=-8$ for $j \geqslant 1$ in Theorem 5. Then $d_{j}=9^{j}-1$ for $j \geqslant 1$ and we can take $\rho_{x}=1$ and $\rho_{0}=9$. With the same notation as before,

$$
\Delta_{n} f=-\frac{1}{8} \sum_{m=1}^{\infty} a_{m}\{\hat{f}(m n)+\hat{f}(-m n)\}
$$

gives the errors for the approximate integrals obtained by cubic interpolation.

(c) Take $q=2, c_{0}=1, c_{1}=-3$ and $c_{j}=45$ for $j \geqslant 2$ in Theorem 5. Then

$$
d_{j}=\operatorname{im}\{(2+\sqrt{ }-44)(1+\sqrt{ }-44) / \sqrt{ } 44\}
$$

for $j \geqslant 1$ and we can take $\rho_{x}=1$ and $\rho_{0}=4 \sqrt{ } 3$. In this case

$$
\Delta_{n} f=\frac{1}{45} \sum_{m=1}^{\infty} a_{m}\{\hat{f}(m n)+\hat{f}(-m n)\}
$$

gives the errors for the approximate integrals defined by Boole's formula.

\section{References}

P. Bachman (1894), Die Analytische Zahlentheorie (Leipzig).

R. Dedekind (1857), 'Abrifs einer Theorie der höhern Congruenzen in Bezug auf einer reellen PrimzahlModulus', J. reine angew. Math. 54, 1-26.

L. Euler (1748), Introductio in analysin infinitorum, Vol. I (Lausanne).

G. H. Hardy (1949), Divergent series (Oxford).

E. Hille (1937), 'The inversion problem of Möbius', Duke Math. J. 3, 549-569.

E. Hille and O. Szasz (1936), 'On the completeness of Lambert functions', Bull. Amer. Math. Soc. 42, 411-418. II. Ann. of Math. 37, 801-815.

C. Jordan (1960), Calculus of finite differences (Dover reprint).

E. Landau (1899), 'Contribution à la théorie de la fonction $\zeta(s)$ de Riemann', C. R. Acad. Sci. Paris, Sér. $A-B$ 129, 812-815

J. Liouville (1857), 'Sur l'expression $\phi(n)$, qui marque combien la suite $1,2,3, \ldots, n$ contient de nombres premiers à $n^{\prime}, J$. de Math. (2) 2, 110-112.

J. H. Loxton and J. W. Sanders (1980), 'The kernel of a rule of approximate integration', J. Austral. Math. Soc. (Ser. B) 21, 257-267.

J. N. Lyness (1970), 'The calculation of fourier coefficients by the Möbius inversion of the Poisson summation formula. Part I. Functions whose early derivatives are continuous', Math. Comp. 24, 101-135.

A. F. Möbius (1832), 'Ueber einer besondere Art von Umkehrung der Reihen', J. reine angew. Math. 9, $105-123$.

B. Riemann (1859), 'Ueber die Anzahl der Primzahlen unter einer gegebenen Grösse', Monatsberichte der Berliner Akademie, 671-680.

G.-C. Rota (1964), 'On the foundations of combinatorial theory I. Theory of Möbius functions', $Z$. Wahrscheinlichkeitstheorie und Verw. Gebiete 2, 340-368. 
P. L. Tchebychef (1851), 'Note sur différentes séries', J. de Math. (1) 16, 337-346.

P. L. Tchebychef (1852), 'Mémoire sur les nombres premiers', J. de Math. (1) 17, 366-390.

A. Wintner (1943), Eratosthenian averages (Baltimore).

School of Mathematics

University of New South Wales

Kensington, N.S.W. 2033

Australia 